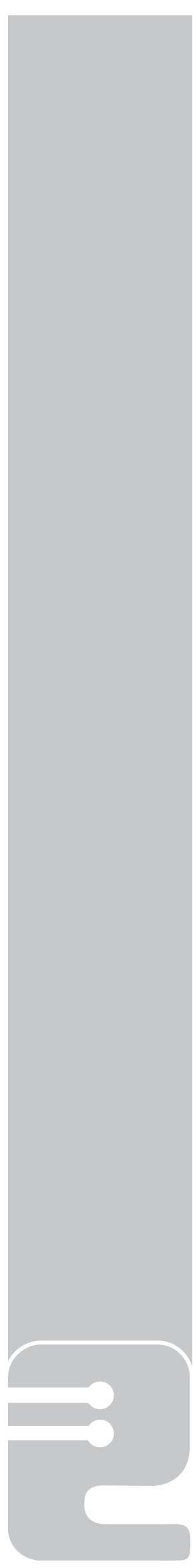

\title{
Perspectiva de las competencias genéricas en ingeniería
}

\author{
Luz Stella Torres Acevedo ${ }^{1}$
}

Recibido: 2 de marzo de 2015 Aprobado: 25 de mayo de 2015

\section{Resumen}

Los requerimientos a los profesionales de la ingeniería cada vez son mayores. El conocimiento evoluciona rápidamente y los futuros profesionales deben adquirir capacidades que les permitan adaptarse a los cambios. Tomando como referencia el Proyecto Tuning Europa y América Latina y algunos perfiles deseados en los profesionales de ingeniería, formulados por destacados estamentos nacionales e internacionales, se evidencia la necesidad de fortalecer las competencias genéricas en los currículos de ingeniería. Adicionalmente, se analizó el desempeño de los estudiantes de la Facultad de Ingeniería de UNIMINUTO en competencias genéricas en las pruebas de Estado de 2012-1 a 2013-2.

\section{Palabras claves}

Competencias genéricas, Proyecto Tuning, ingeniería, desempeño profesional.

\section{Abstract}

The requirements for Engineering professionals are increasing, the knowledge changes and evolves so quickly that future professionals must acquire skills that enable them to adapt to these changes. Taking the Tuning Project Reference As Europe and Latin America and some desired profiles Engineering professionals, made by prominent national and international estates is achieved demonstrate the need to strengthen generic competencies in curricula of engineering, also analysis of the performance of students of the faculty of engineering in generic skills tests 1-2012 to 2-2013 state.

\section{Keywords}

Generics competences, Tuning Project, engineering, professional profile.

\footnotetext{
1 Ingeniero electrónico de la Universidad Pedagógica y Tecnológica de Colombia, especialista en Pedagogía y Docencia Universitaria de la Universidad La Gran Colombia, candidata a magíster en Administración y Planificación Educativa de la Universidad Metropolitana de Ciencia y Tecnología de Panamá. Docente instructor de Tecnología en Electrónica UNIMINUTO Luz.torres@uniminuto.edu.
} 


\section{INTRODUCCIÓN}

La formación por competencias se ha planteado desde los años setenta pero no fue sino hasta el final del siglo XX que tomó fuerza con el surgimiento de iniciativas como el Proyecto Tuning Europa. Éste es un esfuerzo de más de 100 universidades europeas por robustecer la formación superior, que ha logrado consolidar algunas directrices de formación. El proyecto en la fase uno es un documento detallado de la necesidad de las competencias genéricas en la educación superior, que ha dado como resultado la estandarización de 30 competencias necesarias en la formación profesional. Una revisión de este mismo proyecto en el continente dio origen al Proyecto Alfa-Tuning en América Latina, el cual mantuvo 22 competencias iguales al proyecto europeo, incluyó y reagrupó otras, y definió 27 competencias genéricas (Proyecto Tuning América Latina, 2007).

Paralelo a esto, en 2008 el Ministerio de Educación Nacional de Colombia convocó a un grupo de académicos expertos con el objetivo de determinar el conjunto de competencias genéricas que se ajusten a las necesidades del contexto social y cultural. En la reunión se definieron cuatro competencias genéricas pertinentes: comunicación en lengua materna y otra lengua, pensamiento matemático, ciudadanía y manejo de tecnologías de la información y la comunicación (TIC) (Ministerio de Educación Nacional, 2009).

En 2009, el Decreto 3963 reglamentó el examen de Estado para la educación superior e inició la evaluación de competencias a los egresados de todas las carreras de pregrado. Entre las competencias por evaluar se encontraban las genéricas, que integran cuatro aspectos: comunicación escrita, razonamiento cuantitativo, lectura crítica, competencias ciudadanas e inglés (Icfes, 2014).

Teniendo como referente el Proyecto Tuning y los perfiles de ingenieros de importantes organismos internacionales y nacionales, esta reflexión tiene como objetivo dar a conocer al lector los argumentos para responder a la pregunta ¿Realmente las competencias genéricas son indispensables en la vida profesional de cualquier ingeniero?

\section{DISCUSIÓN}

\subsection{Competencias en educación superior}

En la actualidad, el enfoque de formación basado en competencias se ha generalizado en el ámbito de la educación superior y por ende en el laboral. Según Sanz (2010), "El constructo competencia ha alcanzado gran importancia en la última década y es utilizado tanto en la legislación profesional como en la educativa". Este autor también destaca el hecho de que la Unión Europea haya hecho acuerdos en torno a la formación por competencias para reforzar y estandarizar la educación, facilitándoles a los egresados su inclusión al mundo laboral. Entre estos esfuerzos se destaca el Proyecto Tuning Europa, según el cual las competencias se pueden dividir en dos: genéricas y específicas. Las primeras son aquellas que debe adquirir cualquier profesional, mientras que las segundas son las relativas a cada profesión.

Sumado a lo anterior, el informe de la Unesco, emanado de la Comisión Internacional sobre la Educación para el Siglo XXI, presidida por Jacques Delors, enuncia que la educación a lo largo de la vida se basa en cuatro pilares:

1. aprender a aprender, lo que genera autonomía y capacidad de formarse con responsabilidad, aprovechando las posibilidades que ofrece la educación a lo largo de la vida;

2. aprender a hacer, que permite formar al ser humano para que cumpla una función social que lo posibilite para actuar frente a un gran número de situaciones y trabajar en equipo;

3. aprender a ser, para desarrollar la personalidad y para que el individuo pueda obrar con autonomía y responsabilidad personal;

4. aprender a convivir, como posibilidad de construir, reconstruir y transformar la sociedad por medio del contacto con el otro, respetando los valores del pluralismo, comprensión mutua y paz, e incrementando la posibilidad de compartir e interactuar con los demás.

Estos pilares se pueden ver reflejados como competencias instrumentales, sistémicas e interpersonales, mejor conocidas como genéricas.

Así, pues, el Proyecto Tuning es uno de los más amplios y concienzudos estudios acerca de las competencias genéricas. Nació como una iniciativa de más de 100 universidades europeas para generar estándares que propendieran a la calidad de la educación superior en Europa. En una primera fase, Tuning propuso 30 competencias genéricas divididas en tres grupos: sistémicas, interpersonales e instrumentales. Cuatro años más tarde, América Latina se suma a la iniciativa europea, dando origen al Proyecto Alfa-Tuning América Latina, en el que en una primera etapa se revisaron las competencias genéricas propuestas por Europa, se propusieron nuevas y finalmente se lograron definir 27 (tabla 1). 


\begin{tabular}{|c|c|c|}
\hline COMPETENCIAS & TUNING EUROPA & TUNING AMÉRICA LATINA \\
\hline INSTRUMENTALES & $\begin{array}{l}\text { Capacidad de análisis y síntesis } \\
\text { Capacidad de organizar y planificar } \\
\text { Conocimiento generales básicos de la } \\
\text { profesión } \\
\text { Comunicación oral y escrita en la propia } \\
\text { lengua } \\
\text { Conocimientos de una segunda lengua } \\
\text { Capacidades básicas del manejo del } \\
\text { ordenador } \\
\text { Habilidades de gestión de la información } \\
\text { Resolución de problemas } \\
\text { Toma de decisiones }\end{array}$ & $\begin{array}{l}\text { Capacidad de abstracción, análisis y síntesis } \\
\text { Capacidad para organizar y planificar el tiempo } \\
\text { Conocimientos sobre el área de estudio y la } \\
\text { profesión } \\
\text { Capacidad de comunicación oral y escrita } \\
\text { Capacidad de comunicación en un segundo } \\
\text { idioma } \\
\text { Habilidades en el uso de las tecnologías de la } \\
\text { información y la comunicación } \\
\text { Habilidades para buscar, procesar y analizar } \\
\text { información procedente de fuentes diversas } \\
\text { Capacidad para tomar decisiones } \\
\text { Capacidad para identificar, plantear y resolver } \\
\text { problemas }\end{array}$ \\
\hline INTERPERSONALES & $\begin{array}{l}\text { Trabajo en equipo } \\
\text { Capacidad crítica y autocrítica } \\
\text { Habilidades en las relaciones interpersonales } \\
\text { Capacidad de trabajar en un equipo } \\
\text { interdisciplinar } \\
\text { Capacidad de comunicarse con expertos de } \\
\text { otras áreas } \\
\text { Reconocimiento a la diversidad y la } \\
\text { multiculturalidad } \\
\text { Habilidad de trabajar en un contexto } \\
\text { internacional } \\
\text { Compromiso ético }\end{array}$ & $\begin{array}{l}\text { Capacidad crítica y autocrítica } \\
\text { Capacidad de trabajo en equipo } \\
\text { Habilidades interpersonales } \\
\text { Habilidad para trabajar en contextos } \\
\text { internacionales } \\
\text { Capacidad de motivar y conducir hacia metas } \\
\text { comunes } \\
\text { Compromiso con la preservación del medio } \\
\text { ambiente } \\
\text { Compromiso con su medio sociocultural } \\
\text { Valoración y respeto por la diversidad y } \\
\text { multiculturalidad } \\
\text { Compromiso ético } \\
\text { Responsabilidad social y compromiso ciudadano }\end{array}$ \\
\hline SISTÉMICAS & $\begin{array}{l}\text { Capacidad de aplicar los conocimientos en la } \\
\text { práctica } \\
\text { Habilidades de investigación } \\
\text { Capacidad de aprender } \\
\text { Capacidad de adaptación a nuevas } \\
\text { situaciones } \\
\text { Capacidad de generar nuevas ideas } \\
\text { (creatividad) } \\
\text { Liderazgo } \\
\text { Conocimiento de culturas y costumbres de } \\
\text { otros países } \\
\text { Habilidad para trabajar de forma autónoma } \\
\text { Diseño y gestión de proyectos } \\
\text { Iniciativa y espíritu emprendedor } \\
\text { Preocupación por la calidad } \\
\text { Motivación de logro }\end{array}$ & $\begin{array}{l}\text { Capacidad de aplicar los conocimientos en la } \\
\text { práctica } \\
\text { Capacidad de investigación } \\
\text { Capacidad de aprender y actualizarse } \\
\text { permanentemente } \\
\text { Capacidad de actuar en nuevas situaciones } \\
\text { Capacidad creativa } \\
\text { Liderazgo } \\
\text { Habilidad para trabajar de forma autónoma } \\
\text { Capacidad para formular y gestionar proyectos } \\
\text { Iniciativa y espíritu emprendedor } \\
\text { Compromiso con la calidad }\end{array}$ \\
\hline
\end{tabular}

\subsection{La ingeniería y las competencias genéricas}

¿Cuáles deben ser las competencias genéricas desarrolladas por un ingeniero durante su formación para tener un graduado con un perfil adecuado al siglo XXI? Es una pregunta que preocupa a estamentos tanto estatales como privados. "La evaluación de las competencias necesarias para los egresados de ingeniería son aspectos que llevan mucho tiempo en discusión, tanto estamentos gubernamentales como privados se han preocupado por la calidad de los profesionales en las áreas de ingeniería" (Martín, 2005). 
Como resultado de esta preocupación, la Higher Engineering Education for Europe $\left(\mathrm{H}_{3} \mathrm{E}\right)$ realizó un importante estudio con el objetivo de determinar la calidad de la educación en áreas de ingeniería en Europa, creando un grupo denominado WG2, Quality and Recongnition in Engineering Education", que fijó 21 ítem como lineamientos para la acreditación de programas universitarios en ingeniera en Europa:

1. Apropiado conocimiento de matemáticas y ciencias, y habilidad para aplicarlos con efectividad en los problemas de ingeniería.

2. Conocimiento de la práctica técnica industrial.

3. Conocimiento de las materias teóricas relevantes en ingeniería y habilidad para aplicarlos con efectividad a los problemas de ingeniería.

4. Conocimiento de la práctica industrial de la ingeniería.

5. Conocimiento interdisciplinario y habilidad para aplicarlo con efectividad en los problemas de ingeniería.

6. Conocimiento del impacto de las soluciones de ingeniería en un contexto global y social.

7. Competencia en investigación y desarrollo dentro de la ingeniería.

8. Destreza y habilidad directiva.

9. Dominar el inglés como lengua de trabajo profesional y medio de comunicación del ingeniero.

10. Habilidad para trabajar en equipo.

11. Habilidad para comunicar con efectividad.

12. Habilidad para documentarse con efectividad.

13. Habilidad para trabajar, comunicar y cooperar en un entorno internacional.

14. Comprensión crítica.

15. Comprensión sistemática y enfoque holístico que le permita considerar, y después actuar en consecuencia, la relación entre su actividad en la ingeniería y otros campos.

16. Conocimiento de la responsabilidad ética y profesional.

17. Entendimiento del impacto de las soluciones de ingeniería en un contexto global y social basado en una educación generalista.

18. Compromiso del cambio hacia una sociedad del desarrollo sostenible (o conocimiento del desarrollo sostenible).
19. Visión empresarial.

20. Conciencia de la necesidad de aprender a lo largo de la vida y habilidad para hacerlo.

21. Participación comprometida en la definición de políticas tecnológicas y económicas.

Al observar las habilidades definidas por WG2 se puede afirmar que muchas están directamente relacionadas con la adquisición de competencias de tipo instrumental, interpersonal y sistémico, aplicadas a un área específica de la ingeniería, lo cual confronta la necesidad de reformular currículos que favorezcan la adquisición de dichas competencias.

Sumado a esto, la Acreditation Board for Engineering and Technology (ABET), organización no gubernamental norteamericana, sin ánimo de lucro, que trabaja para acreditar la calidad de la educación en ingeniería y tecnología en 23 países, también formuló 11 competencias indispensables que deben tener los egresados en áreas de ingeniería:

1. Capacidad de aplicar el conocimiento de las matemáticas, la ciencia y la ingeniería.

2. Capacidad de diseñar y conducir experimentos, así como de analizar e interpretar datos.

3. Capacidad para diseñar sistemas, componentes o procesos de acuerdo con las necesidades y dentro de restricciones reales de tipo técnico, económico, ambiental, social, político, ético, y de salud y seguridad.

4. Capacidad para trabajar en equipos multidisciplinarios.

5. Capacidad de identificar, formular y solucionar problemas de ingeniería.

6. Comprensión de la responsabilidad profesional y ética.

7. Capacidad para comunicarse de manera eficiente.

8. Educación amplia necesaria para entender el impacto de las soluciones de la ingeniería en un contexto global tanto social como económico y ambiental.

9. Capacidad y reconocimiento de la necesidad de mantener una actitud de aprendizaje continuo a lo largo de la vida.

10. Conocimiento actualizado de los temas contemporáneos.

11. Capacidad para utilizar las técnicas, habilidades y herramientas modernas de la ingeniería necesarias para la práctica de la ingeniería. 
Por otro lado, en busca de unas competencias adaptadas al mundo moderno, el Instituto Tecnológico de Massachusetts (MIT), una de las instituciones más reconocidos a escala mundial por sus altos estándares académicos en las áreas de ingeniería, plantea la propuesta Conceive, Desing, Implement and Operate (CDIO), que formula las competencias que a su criterio requieren los nuevos profesionales de ingeniería:

\section{Conocimiento técnico y razonamiento}

- Demostrar capacidad para usar los principios de las ciencias básicas.

- Aplicar los principios de las ciencias de la ingeniería.

- Demostrar capacidad para aplicar el conocimiento de las áreas profesionales de la ingeniería.

- Habilidades y atributos personales y profesionales

- Analizar y resolver problemas de ingeniería.

- Orientar investigación y experimentos sobre problemas de ingeniería.

- Pensamiento sistémico.

- Demostrar habilidades personales que contribuyan al éxito de la práctica de la ingeniería.

- Demostrar habilidades profesionales que contribuyan al éxito de la práctica de la ingeniería.

- Habilidades interpersonales.

- Liderar y trabajar en grupos.

- Comunicación efectiva.

- Comunicación en idiomas extranjeros.

- Reconocer la importancia del contexto social en la práctica de la ingeniería.

- Apreciar diferentes culturas empresariales y trabajar exitosamente en organizaciones.

- Concebir y desarrollar sistemas de ingeniería.

- Diseñar sistemas complejos.

- Implementar procesos de hardware y software y gestionar los proyectos de implementación.

- Operar sistemas complejos, procesar y gestionar operaciones.
Aunque son evidentes las diferencias entre las competencias formuladas por ABET y CDIO, también se pueden observar algunas coincidencias como liderazgo, comunicaciones interpersonales, trabajo en equipo, capacidad de análisis y de comunicación, entre otras, habilidades directamente relacionadas con las competencias genéricas y aportantes a un perfil de ingeniero dinámico, con capacidad de autoaprendizaje, de adaptación a los cambios, con competencias que les permitan apropiarse del conocimiento, más allá de una aula de clase, como artífice de cambios tecnológicos y sociales.

Colombia no es ajena a los retos laborales y sociales que el siglo XXI les trae a los nuevos profesionales en ingeniería. Por esto, en el año 2004 el decano de una de las principales facultades de ingeniera del país, junto con un grupo de académicos, formuló el perfil del ingeniero del futuro: "Un ingeniero capaz de identificar y entender los problemas de su tiempo, de interpretar las necesidades sociales y de responder a ellas con soluciones fundadas en la comprensión y el dominio de las ciencias, las matemáticas, la tecnología y los métodos de la ingeniería. Un ingeniero que se constituya en líder y guía de la sociedad en que se desenvuelva en lo que respecta a la identificación, apropiación, uso y generación de la tecnología que mejor contribuya a su avance sostenible. Un ingeniero que sea un líder en la creación e impulso de empresas de base tecnológica y cuyo liderazgo contribuya al desarrollo empresarial del país” (Hernández, 2004).

En el perfil formulado por Hernández se evidencian aspectos como liderazgo, capacidad de aprender, compromiso ético y social, como requerimientos para un profesional en ingeniería. Se puede afirmar que desde 2004 ya se estaba hablando de la necesidad de las competencias genéricas como elemento fundamental para lograr un perfil profesional adecuado a los requerimientos solicitados por el sector laboral y, en general, por la sociedad.

Sumado a esto, en 2007 la Asociación Colombiana de Facultades de Ingeniería (Acofi) publicó las memorias del foro "El Ingeniero Colombiano para el año 2020, retos para su formación", preparatorio del XXVI encuentro que tenía como objetivos identificar factores y plantear estrategias que fortalecieran la formación de los ingenieros a 2020. En el segundo foro que tuvo lugar el 23 de febrero de 2006 en el aula magistral de la Universidad del Norte de Barranquilla, se concluyó que las competencias solicitadas para los ingenieros deberían ser:

- Ser integral.

- Responsabilidad social para ubicar los conocimientos en su país y no ser pasivos frente a las necesidades sociales 
- Educación permanente.

- Formación de posgrado.

- Capacidad para ser autónomo, y aprender y desaprender.

- Que “crea en lo nuestro”.

- Capacidad de liderar y proponer cambios, no sólo soluciones a necesidades.

- Capacidad de innovar y crear productos y tecnología.

- Actitud positiva frente a la sociedad y la vida.

- Capacidad para utilizar el conocimiento y la experiencia de otros países adaptándolos al propio.

- Disposición para aprovechar los recursos para incrementar la economía en la sociedad.

En el marco de este mismo encuentro, en el quinto foro llevado a cabo en la ciudad de Tunja, en la conferencia "El perfil del ingeniero del año 2020", ofrecida por el ingeniero Joaquín Oramas, vicerrector académico de la Universidad Autónoma de Occidente, se describieron los nuevos retos del ingeniero colombiano y se planteó un perfil para formarlo (Acofi, 2007):

- Crear ambientes en los que el diseño y la promoción de actividades y situaciones de aprendizaje propicien el desarrollo del potencial intelectual de los individuos.

- Desarrollar la capacidad para enfrentar la realidad de forma reflexiva, crítica y constructiva, con grandes dosis de autonomía y autodeterminación.

- Identificar plenamente los ejes transversales que afectan las situaciones más problemáticas o socialmente relevantes de la zona, lugar, país, región, o del mundo.

- Desarrollar la capacidad de enfrentar los problemas con el ingenio propio de los ingenieros y la habilidad de actuar de manera interrelacionada e interdisciplinaria.

- Adquirir la capacidad de aprender a aprender que les permita afrontar con éxito la situación cambiante de la realidad y adaptarse rápidamente, y de modo innovador, a las problemáticas que se les presenten. Para desempeñarse eficientemente en su trabajo, más que requerir un gran número de características o un enorme repertorio de habilidades específicas, los ingenieros deberán tener la capacidad de adquirir nuevo conocimiento para resolver problemas, así como emplear la creatividad y el pensamiento crítico en el diseño de formas diferentes de aproximación a los problemas existentes.

Este perfil de formación hace énfasis en graduar ingenieros, que además de tener amplios conocimientos de su área específica sean capaces de liderar procesos productivos que apoyen el crecimiento social y económico del país.

Un año después, en 2008, el Ministerio de Educación Nacional convocó a un grupo de académicos expertos con el objetivo de determinar el conjunto de competencias genéricas que se ajustaran a las necesidades del contexto social y cultural del país. En esta convocatoria se determinaron cuatro competencias genéricas pertinentes: comunicación en lengua materna y otra internacional, pensamiento matemático, ciudadanía y manejo de tecnologías de la información y la comunicación (TICS) (Ministerio de Educación Nacional, 2009). Como consecuencia, el Gobierno colombiano expidió en 2009 el Decreto 3963, que reglamenta el examen de Estado para la educación superior y da inicio a la evaluación de competencias a los egresados de todas las carreras de pregrado. Entre las competencias por evaluar se encontraban las genéricas, que actualmente se evalúan en cuatro aspectos: comunicación escrita, razonamiento cuantitativo, lectura crítica, competencias ciudadanas e inglés (Icfes, 2014).

2.3 ¿Cómo están las competencias genéricas en la facultad de ingeniería?

Luego de hacer un recorrido sobre los perfiles profesionales propuestos por organismos nacionales e internacionales, el ingeniero del nuevo siglo y la importancia que juegan las competencias genéricas en él, se revisará el desempeño de los estudiantes de la Facultad de Ingeniería sede principal, en cuanto a las competencias genéricas evaluadas por la pruebas de Estado Saber Pro en los periodos académicos comprendidos entre 2012-1 a 2013-2.

Las gráficas 1, 2, 3 y 4 corresponden al resultado promedio obtenido por los estudiantes de la sede principal UNIMINUTO, discriminados por facultades y tipo de competencia evaluada. En la gráfica 1 se puede ver que para la Facultad de Ingeniería todas las competencias genéricas evaluadas presentaron valores muy cercanos a los promedios nacionales que, comparados con el desempeño en aspectos como lectura crítica, inglés, escritura y competencias ciudadanas, resultan muy desalentadores. 


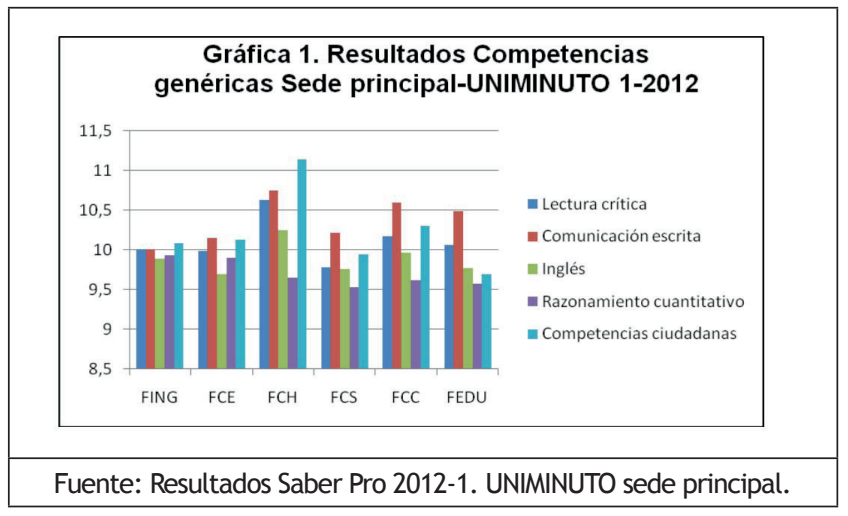

En el periodo 2012-2, gráfica 2 se observa una mejora en el desempeño de los estudiantes, en aspectos como comunicación escrita, inglés y razonamiento cuantitativo, que superan los promedios nacionales, aunque competencias como lectura crítica y competencias ciudadanas tuvieron promedios por debajo del promedio nacional. Además, en la prueba de comunicación escrita el peor desempeño en ese periodo correspondió a la Facultad de Ingeniería.

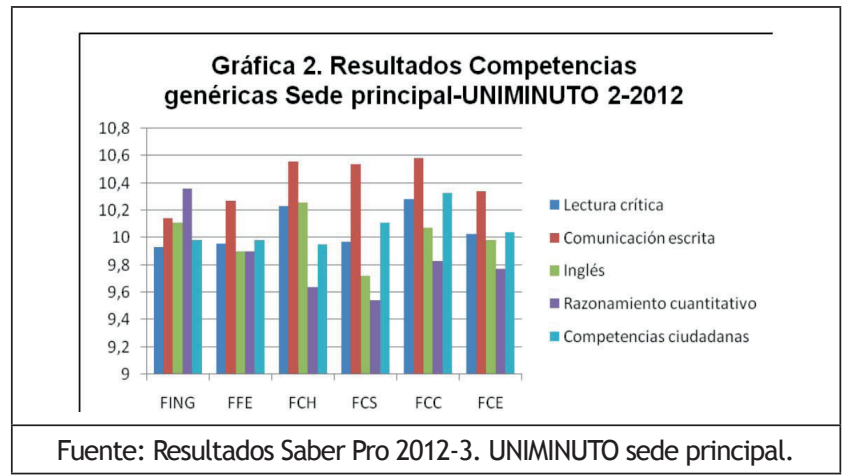

En el primer periodo de 2013, las competencias están muy cercanas a los promedios nacionales. Se destaca razonamiento cuantitativo y nuevamente comunicación escrita, además de no alcanzar los promedios nacionales, presenta el peor desempeño en comparación las demás facultades de la sede como aparece en la gráfica 3.

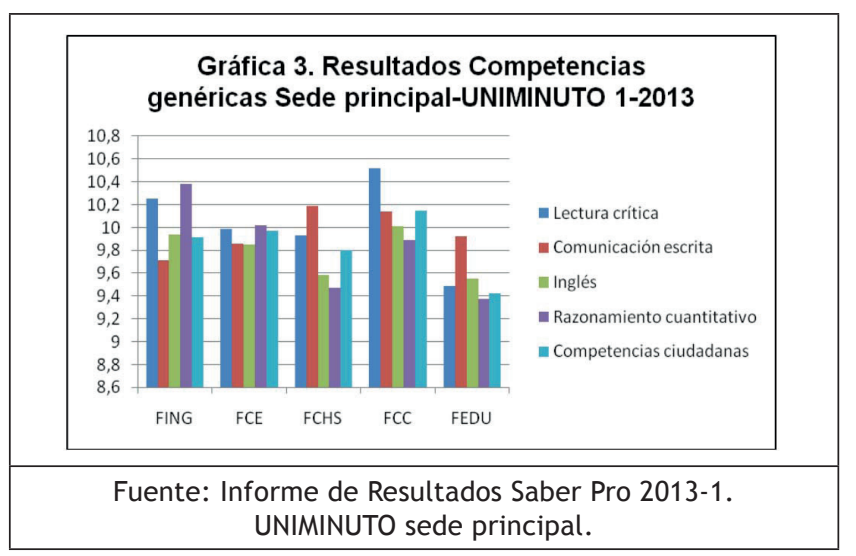

Por último, en el periodo 2013-2 la competencia de inglés está por encima de la media nacional; competencias como lectura crítica, competencias ciudadanas y razonamiento tienen valores muy cercanos a la media nacional, pero otra vez aparece la competencia de comunicación escrita como la de peor desempeño entre todas las pruebas en la facultad, como al comparar los promedios obtenidos en las demás facultades de la sede en la misma prueba, como se observa en la gráfica 4 .

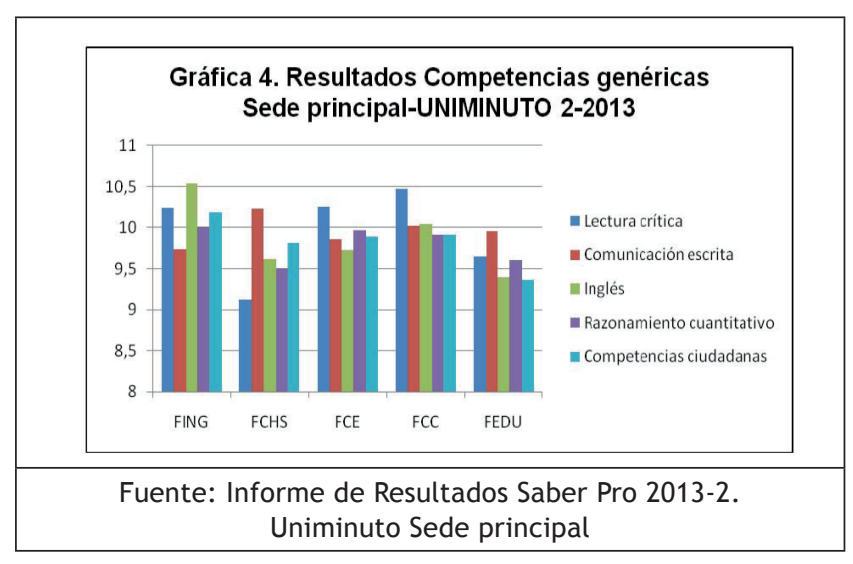

En la gráfica 5 se presenta un consolidado para la facultad, en la que se muestra la evolución de las competencias en cuatro periodos académicos. Como se enunció, el desempeño en las pruebas Saber Pro en la Facultad de Ingeniera no es destacable, los resultados obtenidos en la mayoría de casos están muy cercanos a la media nacional y, si bien es cierto que se tuvo un leve aumento en el promedio en la mayoría de pruebas, la competencia de comunicación escrita presenta serias deficiencias, por cuanto en dos periodos académicos no alcanzó siquiera los estándares nacionales y en todos los periodos académicos la Facultad de Ingeniería tuvo el más bajo promedio de la sede.

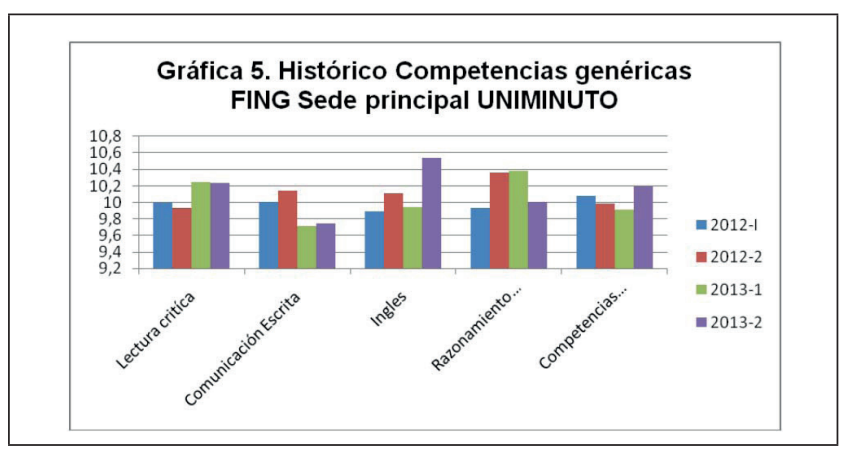

\section{CONCLUSIONES}

La ingeniería es una disciplina en la cual se ha dado importancia especialmente a la apropiación del conocimiento y su aplicación centrado en el "saber 
hacer", restándole valor a las capacidades que involucran aprender a ser, convivir y aprender a aprender, tres pilares fundamentales de la educación, según la Unesco. Esta nueva visión de conocimiento da como resultado la necesidad de revisar y proponer un cambio en los currículos de ingeniería, que le permitan al estudiante adquirir competencias que, además de formarlo en una disciplina específica, le permita tener herramientas para desenvolverse en cualquier aspecto de la vida.

Las competencias genéricas forman parte fundamental del perfil del ingeniero del futuro. Importantes organizaciones de tipo académico no sólo en Colombia sino a escala mundial, proponen perfiles de ingenieros en los que las competencias interpersonales, sistémicas e instrumentales se evidencian como requerimiento fundamental para un desempeño profesional acorde con las necesidades del mundo moderno.

El desempeño de los estudiantes de la Facultad de Ingeniera en las pruebas Saber Pro, en el módulo de competencias genéricas, tiene valores promedio de desempeño muy cercanos a la media nacional, lo que indica que el ejercicio de los estudiantes apenas si cumple con los mínimos estándares requeridos. Es una situación preocupante desde el punto de vista de calidad de la educación impartida en la facultad, lo que recuerda a Tünnermann (2000): "En el futuro, la calidad de las universidades será juzgada más por la calidad de los alumnos que por la calidad de los profesores".

La competencia de comunicación escrita es visiblemente la de más bajo desempeño en las pruebas, si bien es cierto que por la naturaleza misma de la ingeniería y su afinidad con las ciencias exactas "los estudiantes de ingeniería son propensos a usar ecuaciones y gráficas para expresarse" Valencia (2012). Esto no justifica el hecho de no poder expresarse por escrita eficientemente. Haciendo alusión a los perfiles de ingenieros propuestos por WG2, ABBET y MIT se observa que comunicarse efectivamente forma parte de los requerimientos solicitados en dichos perfiles, así que las necesidad de fortalecer la comunicación escrita en los estudiantes de ingeniería debe convertirse en una prioridad si se desea que el desempeño de nuestros graduados sea destacable en el medio laboral.

\section{REFERENCIAS}

1. Proyecto Tuning América Latina. (2007). Reflexiones y perspectivas de la educación superior en América Latina. Informe final (Proyecto Tuning América Latina, 2004-2007). Universidad de Deusto. Universidad de Groningen.
2. Rivero, L. (1999). Perfil del ingeniero del futuro. Revista de la Escuela Colombia de Ingeniería, 34, pp. 20-24.

3. Ministerio de Educación Nacional. (2009). Revolución Educativa. Educación Superior. Bogotá.

4. Icfes. (Enero de 2014). Recuperado de www. icfes.gov.co/saber-pro/informacion el 10 de julio de 2014.

5. Chávez, U. (1998). Las competencias en la educación para el trabajo. Seminario sobre Formación Profesional y Empleo. México.

6. Sanz, M. L. (2010). Competencias cognitivas en educación superior. Madrid: Nacea S.A.

7. Confedi. (2006). Primer acuerdo sobre competencias genéricas. Tercer Taller sobre Competencias. Bahía Blanca: Cofendi.

8. Hernández, J. T. (2004). Plan de renovación de la Facultad de Ingeniería. Nota Uniandina, 28.

9. Tuning Educational Structures in Europa. (2001). Contribución de las universidades al proceso de Bolonia. Sócrates-Tempus.

10. Jacques Delors. (1996). La educación encierra un tesoro. París: Unesco.

11. Martín, R. (2005). Modelo para la mejora de la calidad de la formación en ingeniería mecánica aplicado a la ingeniería industrial. Universidad de Cádiz, Ingeniería. Cádiz: Servicio de Publicaciones de la Universidad de Cádiz.

12. Acofi. (2007). Foro "El ingeniero colombiano del año 2020. Retos para su formación." Segundo foro, pp. 297. Bogotá: Acofi.

13. Valencia, A. (2012). El problema de la comunicación en ingeniería: el caso de las universidades. Ingeniería y sociedad, pp. 39-45.

14. Tünenermann, C. (2000). Universidad y sociedad: balance histórico y perspectivas en Latinoamérica. Caracas: Universidad Central de Venezuela.

15. Martín, R. (2005). Modelo para la mejora de la calidad de la formación en ingeniería mecánica. Universidad de Cádiz. Servicio de publicaciones de la Universidad de Cádiz. 